\title{
Convection-enhanced delivery of sorafenib and suppression of tumor progression in a murine model of brain melanoma through the inhibition of signal transducer and activator of transcription 3
}

\author{
Zhaoxia Zou, MD, MS, ${ }^{1}$ Yufang Yin, MS, ${ }^{1}$ Jenny Lin, BA, ${ }^{1}$ Li-chen J. Hsu, MS, ${ }^{1}$ \\ Vanessa L. Brandon, ${ }^{1}$ Fan Yang, PhD, ${ }^{1}$ Richard Jove, PhD, ${ }^{1}$ Rahul Jandial, MD, PhD, ${ }^{1}$ \\ Gang Li, PhD, ${ }^{2}$ and Mike Y. Chen, MD, PhD ${ }^{1}$ \\ 'Division of Neurosurgery, Department of Surgery, City of Hope National Medical Center, Duarte, California; and ${ }^{2}$ Faculty of \\ Health Sciences, University of Macau, China
}

\begin{abstract}
OBJECTIVE Despite recent advances, metastatic melanoma remains a terminal disease, in which life-threatening brain metastasis occurs in approximately half of patients. Sorafenib is a multikinase inhibitor that induces apoptosis of melanoma cells in vitro. However, systemic administration has been ineffective because adequate tissue concentrations cannot be achieved. This study investigated if convection-enhanced delivery (CED) of sorafenib would enhance tumor control and survival via inhibition of the signal transducer and activator of transcription 3 (Stat3) pathway in a murine model of metastatic brain melanoma.

METHODS Melanoma cells treated with sorafenib in vitro were examined for signaling and survival changes. The effect of sorafenib given by CED was assessed by bioluminescent imaging and animal survival.

RESULTS The results showed that sorafenib induced cell death in the 4 established melanoma cell lines and in 1 primary cultured melanoma cell line. Sorafenib inhibited Stat3 phosphorylation in HTB65, WYC1, and B16 cells. Accordingly, sorafenib treatment also decreased expression of Mcl-1 mRNA in melanoma cell lines. Because sorafenib targets multiple pathways, the present study demonstrated the contribution of the Stat3 pathway by showing that mouse embryonic fibroblast (MEF) Stat3 +/+ cells were significantly more sensitive to sorafenib than MEF Stat3 $-/$ - cells. In the murine model of melanoma brain metastasis used in this study, CED of sorafenib increased survival by $150 \%$ in the treatment group compared with animals receiving the vehicle control $(p<0.01)$. CED of sorafenib also significantly abrogated tumor growth.
\end{abstract}

CONCLUSIONS The data from this study indicate that local delivery of sorafenib effectively controls brain melanoma. These findings validate further investigation of the use of CED to distribute molecularly targeted agents.

http://thejns.org/doi/abs/10.3171/2015.3.JNS132040

KEY WORDS sorafenib; convection-enhanced delivery; Stat3; melanoma; brain; mice; oncology

$\mathrm{D}$ ESPITE the low incidence of melanoma compared with other cancers, melanoma is the third most common type of cancer to metastasize to the brain. Patients with malignant melanoma have a $37 \%$ chance of being diagnosed with brain metastasis and a $75 \%$ chance of harboring this disease in the brain at autopsy. ${ }^{29}$ When brain metastasis occurs, treatment is extremely difficult due to high resistance to radiation and chemotherapy; median survival with maximal treatment is 5-9 months. Ipilimum- ab, an anticytotoxic T-lymphocyte-associated protein 4 antibody, ${ }^{9}$ and vemurafenib, an inhibitor of mutant B-Raf, ${ }^{20}$ are 2 exciting targeted therapies that have been recently developed. However, due to tumor escape mechanisms, these drugs only extend survival by 2-6 months and, for unclear reasons, ipilimumab and vemurafenib have at most a modest effect on melanoma brain metastasis. ${ }^{7}$

New insights into the biology of this cancer and stateof-the-art drug delivery methods may provide a frame-

ABBREVIATIONS B16-ffluc = firefly luciferase-expressing B16 melanoma cell line; $C E D=$ convection-enhanced delivery; DMSO = dimethyl sulfoxide; IC50 = median inhibitory concentration; IL-6 = interleukin 6; PBS = phosphate-buffered saline; pStat3 = phosphorylation of Stat3; Stat3 = signal transducer and activator of transcription 3. SUBMITTED September 24, 2013. ACCEPTED March 30, 2015.

INCLUDE WHEN CITING Published online November 6, 2015; DOI: 10.3171/2015.3.JNS132040. 
work for novel therapeutic approaches that combine molecular and physical targeting. Of particular interest is signal transducer and activator of transcription 3 (Stat3), an essential signaling molecule in carcinogenesis found to be highly overexpressed in melanomas. ${ }^{36}$ Stat3's transcriptional targets include genes mediating apoptosis (survivin, $B c l-x l, M c l-1$, cellular FLICE-like inhibitory protein); proliferation (c-fos, $c-m y c$, cyclin Dl); invasion (matrix metalloproteinase-2); and angiogenesis (vascular endothelial growth factor). ${ }^{1}$ Functionally, Stat 3 activation has been associated with chemoresistance and radioresistance. ${ }^{1,13}$

Sorafenib was developed as a c-Raf kinase inhibitor and, by extension, was expected to be potent because the Ras-Raf pathway has a prominent role in the biology of melanomas. ${ }^{30}$ Interestingly, our group and Delgado et al. recently demonstrated that sorafenib inhibits Stat3 signaling in medulloblastomas and esophageal carcinoma, respectively. ${ }^{6,35}$ Thus, given its inhibitory targets, sorafenib would seem to be a promising drug for the treatment of melanomas. Indeed, in vitro testing demonstrated good activity. ${ }^{24}$ However, in clinical trials, maximal doses of orally administered sorafenib showed limited activity against metastatic melanoma as a single agent. ${ }^{23}$

The failure of systemically administered sorafenib in clinical trials highlights a challenge of this form of drug delivery. Systemic delivery of therapeutic agents generally results in increased toxicity to normal tissues and organs. Additionally, drug distribution to protected organs, such as the brain, is limited. Hence, there is good rationale for using local delivery methods to target bulky metastasis, which often causes neurological compromise. Accordingly, convection-enhanced delivery (CED), a highly controlled form of microinjection developed for regional drug delivery to the CNS, has received increasing interest due to the potential advantages that it provides when compared with other delivery methods. One is that CED is able to bypass the blood-brain barrier, allowing for targeted delivery into a designated area with limited neurotoxicity. Another potential advantage resides in its capability to distribute therapeutic agents homogeneously over large volumes of normal or pathological tissue, as demonstrated by preclinical and clinical studies., ${ }^{4,15,16,18,19}$ As an aside, nanoparticles as large as viruses have been convected for gene therapy of Parkinson's disease. ${ }^{33}$

In this study, we investigated CED of sorafenib in a murine model of metastatic brain melanoma. We demonstrated that sorafenib in vitro causes cytotoxicity in melanoma cells, at least partially through inhibiting Stat 3 phosphorylation. The results of this study also revealed that CED of sorafenib into melanomas within the brain effectively abrogated tumor growth.

\section{Methods \\ Cell Culture}

The B16 murine melanoma cell line and 3 human melanoma cell lines (A2058, HTB65, and HTB72) were obtained from the American Type Culture Collection. WYC1, a primary melanoma culture, was a generous gift from Dr. James Wang. All cells were maintained in me- dia with $10 \%$ fetal bovine serum and the antibiotics. For B16, WYC1, and A2058 cell lines, Dulbecco's Modified Eagle's Medium (DMEM) was used. For HTB65 and HTB72, Eagle's Minimum Essential Medium (EMEM) was used.

\section{Cell Viability Assay}

Cytotoxicity was determined by CellTiter-Glo Luminescent Cell Viability Assay (Promega). Each well of 96well plates was seeded with 1000 cells in culture medium with $10 \%$ fetal bovine serum. After overnight culture, the cells were treated with different concentrations of sorafenib (Onyx and Bayer Pharmaceuticals) and melphalan. Controls were treated with vehicle control (dimethyl sulfoxide [DMSO]). After 96 hours of treatment, the assay was performed according to the supplier's protocol and measured using a luminescent plate reader. Experiments were performed in quadruplicate and repeated independently at least twice.

\section{Immunoblotting Analysis}

All antibodies were obtained from Cell Signaling. Total protein $(20 \mu \mathrm{g})$ was denatured and separated on $4 \%-12 \%$ gradient Tris-Glycine gels (Invitrogen). After gel electrophoresis, the proteins were transferred to polyvinyl difluoride membranes (Invitrogen). The membranes were blocked for 1 hour at room temperature in blocking buffer (Invitrogen), followed by an overnight incubation at $4{ }^{\circ} \mathrm{C}$ with primary antibodies in $1 \times$ phosphate-buffered saline (PBS) with $0.1 \%$ Tween 20 with 5\% bovine serum albumin. The membranes were then incubated with horseradish peroxidase-labeled antimouse or antirabbit secondary antibodies for 30 minutes at room temperature. Immunoreactivity was detected with SuperSignal West Pico substrate (Pierce).

\section{Firefly Luciferase-Expressing B16 Melanoma Cell Line}

For the animal study, pGL4.51[luc2/cmv/neo] vector (Promega) encoding firefly luciferase was stably transfected into B16 melanoma cells to facilitate noninvasive bioluminescent imaging. Individual stable clones were selected in the presence of $1 \mathrm{mg} / \mathrm{ml} \mathrm{G} 418$ (Sigma).

\section{Sorafenib}

Sorafenib was dissolved in DMSO and Tween 20 at a concentration of $50 \mathrm{mmol} / \mathrm{L}$ and stored at $-20^{\circ} \mathrm{C}$ before use for in vitro and in vivo experiments. For oral administration, sorafenib was freshly prepared in DMSO and Tween 20 at a concentration of $10 \mathrm{mg} / \mathrm{ml}$.

\section{Synergy Calculations}

We used a method described by Hata et al. and Zhang et al. ${ }^{12,39}$ to determine drug synergy. Predicted survival was compared with actual survival values at each dose. Predicted survival (c) was calculated from the equation $\mathrm{c}$ $=(\mathrm{a} \times \mathrm{b}) / 100$, where $\mathrm{a}$ and $\mathrm{b}$ represent survival values for a single agent. The 2 drugs were considered synergistic if the actual survival values were less than $70 \%$ of the predicted survival values. 


\section{B16 Melanoma Xenograft Model}

Six to 8 mice were used for experiments. All animals were housed and handled in accordance with the City of Hope Research Animal Care Committee guidelines. Six- to 8-week-old female C57BL/6 (Charles River Laboratories) mice were anesthetized with an intraperitoneal injection of ketamine and xylazine. Mice were shaved on the head above the nape of the neck. Animals were placed in a Kopf stereotactic frame. A 5-mm skin incision was made along the sagittal suture and a bur hole was drilled into the skull. Firefly luciferase-expressing B16 (B16-ffluc) melanoma cells $\left(1.5 \times 10^{5}\right.$ cells/mouse) suspended in $10 \mu \mathrm{l}$ PBS were stereotactically implanted into the right striatum, $0.5 \mathrm{~mm}$ anterior to and $2.5 \mathrm{~mm}$ to the right of the bregma and $3 \mathrm{~mm}$ below the dural surface. The incision was sealed with Nexaband glue after tumor implantation.

\section{Convection-Enhanced Delivery of Sorafenib}

C57BL/6 mice with B16-ffluc melanoma had sorafenib injected into their striatum at the same incision site using CED. The mice were anesthetized by exposure to $4 \%$ isofluorane for 2 minutes, and then ketamine and xylazine were administered by intraperitoneal injection to maintain anesthesia. The CED system was set up by connecting an injection syringe, which was filled with sorafenib or its carrier, to an external pump with an injecting rate range of $0.03-10 \mu \mathrm{l} / \mathrm{min}$. A 21-gauge needle was used in CED for drug infusion throughout the target region. Animals were placed in a Kopf stereotactic frame and secured using ear bars. A sagittal incision was made and the 21-gauge needle was stereotactically guided to $0.5 \mathrm{~mm}$ anterior to and 2.5 $\mathrm{mm}$ to the right of the bregma, $3 \mathrm{~mm}$ below the brain surface. Delivery was initiated by activating the pump. The delivery rate was $0.2 \mu \mathrm{l} / \mathrm{min}$. After that, the needle was withdrawn, and the incision was glued. For postoperative pain, buprenorphine was subcutaneously administered to provide analgesia.

\section{Animal Experiments and Imaging}

Dose-response curves of CED of varying sorafenib concentrations $(10,100,300,500$, and $1000 \mu \mathrm{M})$ were generated in non-tumor-bearing $\mathrm{C} 57 \mathrm{BL} / 6$ mice to determine the maximal tolerated dose in normal brain. Based on clinical observation and histological analysis (supplemental data), CED of $100 \mu \mathrm{M}$ sorafenib was used for further experiments.

In tumor-bearing animals, CED was performed once or twice after tumor inoculation. In the former group, 10 $\mu \mathrm{l}$ of $100 \mu \mathrm{M}$ sorafenib or vehicle was convected at a rate of $1 \mu \mathrm{l} / \mathrm{min}$ into the brain tumor 7 days after tumor inoculation, using previously described methods. ${ }^{4}$ The same stereotactic coordinates used for tumor implantation were used for CED of the sorafenib. For bioluminescent imaging, anesthetized mice were given $150 \mathrm{mg} / \mathrm{kg}$ Dluciferin intraperitoneally. Five minutes after injection, images were acquired using the Xenogen in vivo imaging system. Bioluminescent signal was measured as photons per second per square centimeter in defined regions of interest using Living Image software (Xenogen). Imaging was performed on Day 6 after tumor implantation immediately prior to treatment, and on Days 1,2, and 3 after CED of the sorafenib. Survival was measured from the time of tumor inoculation. To prevent undue suffering, euthanasia was performed if mice developed significant distress.

In the group in which animals were treated twice, $10 \mu \mathrm{l}$ of $100 \mu \mathrm{M}$ sorafenib was convected on Days 4 and 8 after B16-ffluc injection. Xenogen imaging was performed 2 days after each treatment. In the group in which animals were treated with oral administration of sorafenib, the mice received $50 \mathrm{mg} / \mathrm{kg} /$ day for 7 days by gavage, starting on Day 4 after tumor inoculation.

\section{Statistical Analysis}

One-way ANOVA was used to establish whether significant differences existed between groups. Statistical analyses were performed using SPSS version 14.0 (IBM). A $p<0.05$ was considered statistically significant.

\section{Results}

\section{Sorafenib Cytotoxicity In Vitro}

We first assessed the potency of sorafenib in vitro to confirm its described cytotoxicity. Dose-response curves were generated using 4 established melanoma cell lines (A2058, HTB65, HTB72, and B16) and 1 primary cultured melanoma cell line (WYC1). Toxicity occurred in the micromolar range, with median inhibitory concentrations (IC50) ranging from $4.5 \mu \mathrm{M}$ to $12 \mu \mathrm{M}$ (Fig. 1A, supplementary Fig. 1). At doses approaching $20 \mu \mathrm{M}$, sorafenib was able to cause at least a $90 \%$ reduction in cell viability. These results, showing a relatively high IC50, confirmed our suspicion that systemic delivery would not be able to achieve sufficient concentrations to kill melanoma cells. To treat melanoma, the potency of sorafenib would have to be increased by modifying its pharmacodynamics or pharmacokinetics.

\section{Synergism Between Sorafenib and Melphalan}

Melphalan is a chemotherapeutic agent that has been used in the past to treat melanoma, with modest results. Clinical studies also indicate that systemically administered sorafenib is ineffective. Both drugs have had minimal success in patients. We rationalized that coadministration of sorafenib and melphalan may be synergistic because sorafenib-mediated inhibition of the Ras-Raf kinase pathway has been shown to enhance the chemosensitivity of cancer cells to other chemotherapeutic agents. ${ }^{32}$ While the sorafenib dose was held constant (approximately the IC50 dose), the melphalan dose was increased. Thus, if there was no synergism, then the predicted toxicity at each dose of melphalan would be the additive effect of the sorafenib and the melphalan. With a few minor exceptions, the amount of synergism seen in the HTB65 and WYC1 melanoma cell lines was negligible (Fig. 1B). We also found no evidence of synergy between sorafenib and temozolomide, another drug commonly used to treat melanoma, in A2058, HTB65, HTB72, and WYC1 cells (data not shown). Thus, further experiments used sorafenib alone. 

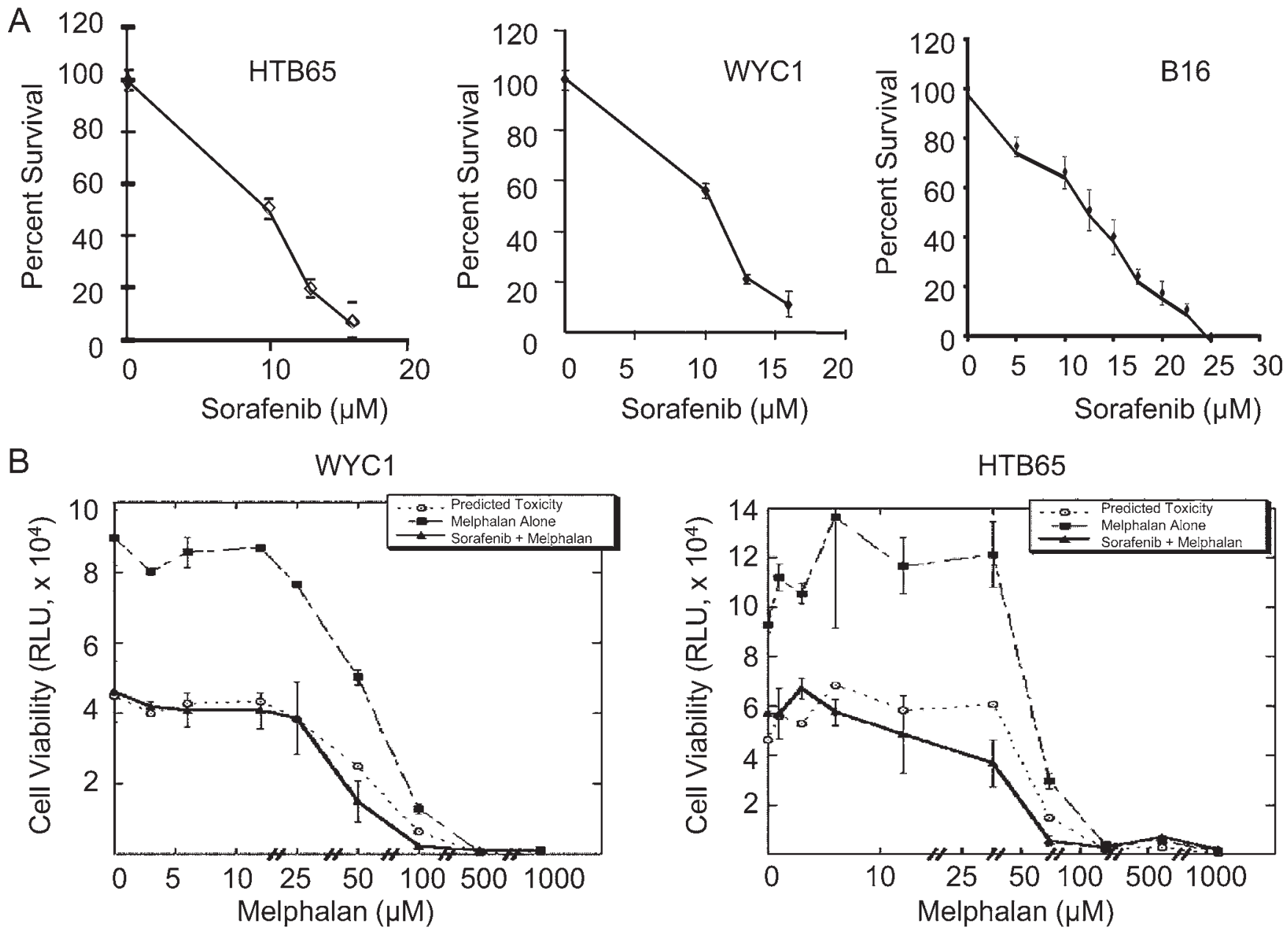

FIG. 1. Sorafenib potency and synergism. The cytotoxicity of sorafenib on melanoma cell lines. Dose-response curves were generated by measuring cell viability 96 hours (HTB65 and WYC1) or 48 hours (B16) after sorafenib treatment (A). Dose-response curves of melphalan alone, melphalan plus sorafenib (10 $\mu \mathrm{M}$; the IC50 dose), and the predicted additive cytotoxicity of melphalan plus sorafenib (B). Significant synergism occurred when survival of melphalan plus sorafenib cells was less than $70 \%$ of the predicted value, an event that seldom occurred.

\section{Sorafenib Inhibits Stat3 Phosphorylation in Melanoma Cell Lines}

Because Stat 3 is highly overexpressed in melanomas and has recently been shown to be a sorafenib target in other cancers, ${ }^{21,31}$ we investigated whether Stat 3 inhibition accounts for at least part of sorafenib's toxicity against melanomas. HTB65, WYC1, and B16 cells were harvested at 5 and 24 hours after sorafenib treatment and then assessed for phosphorylation status of Stat 3 by Western blot (Fig. 2A-C). In HTB65 cells, phosphorylation of Stat3 (pStat3) was only inhibited by sorafenib after 24 hours (Fig. 2A). In WYC1 and B16 cells, pStat 3 was significantly inhibited at both 5 and 24 hours after treatment (Fig. 2B and C).

Interleukin 6 (IL-6) is a strong activator of the Jak/Stat3 pathway, which often occurs through an autocrine loop in cancer cells. A measure of sorafenib's anti-Stat3 activity is whether it can inhibit Stat 3 in the presence of IL-6 stimulation. HTB65 cells were serum starved for 6 hours before treatment with $2 \mu \mathrm{M}$ sorafenib. After 5 or 24 hours of sorafenib exposure, the melanoma cells were subjected to 1 or 4 hours of IL-6 stimulation prior to analysis. Serum starvation abrogated baseline pStat 3 expression (Fig. 2D and E). IL-6 stimulation resulted in pStat 3 expression with or without sorafenib under all conditions. These results indicate that although sorafenib targets Stat3, its suppression of Stat 3 is readily overcome by IL-6.

\section{Sorafenib Preferentially Targets Stat $3+/+$ Cell Lines}

Small-molecule inhibitors often target multiple pathways, making it difficult to determine whether suppression of any particular pathway contributes to a drug's cytotoxicity. To assess the importance of Stat 3 targeting for sorafenib, we examined the cytotoxicity caused by the drug in mouse embryonic fibroblast (MEF) Stat $3+/+$ and MEF Stat3 -/- cells. MEF Stat3 +/+ was significantly more sensitive to both $6 \mu \mathrm{M}$ (Fig. 3A) and $12 \mu \mathrm{M}$ (Fig. $3 B)$ sorafenib $(p<0.05)$. If Stat 3 was not an important sorafenib target, then one would have expected MEF Stat3 $-/-$ cells to have been equally, if not more, sensitive. 


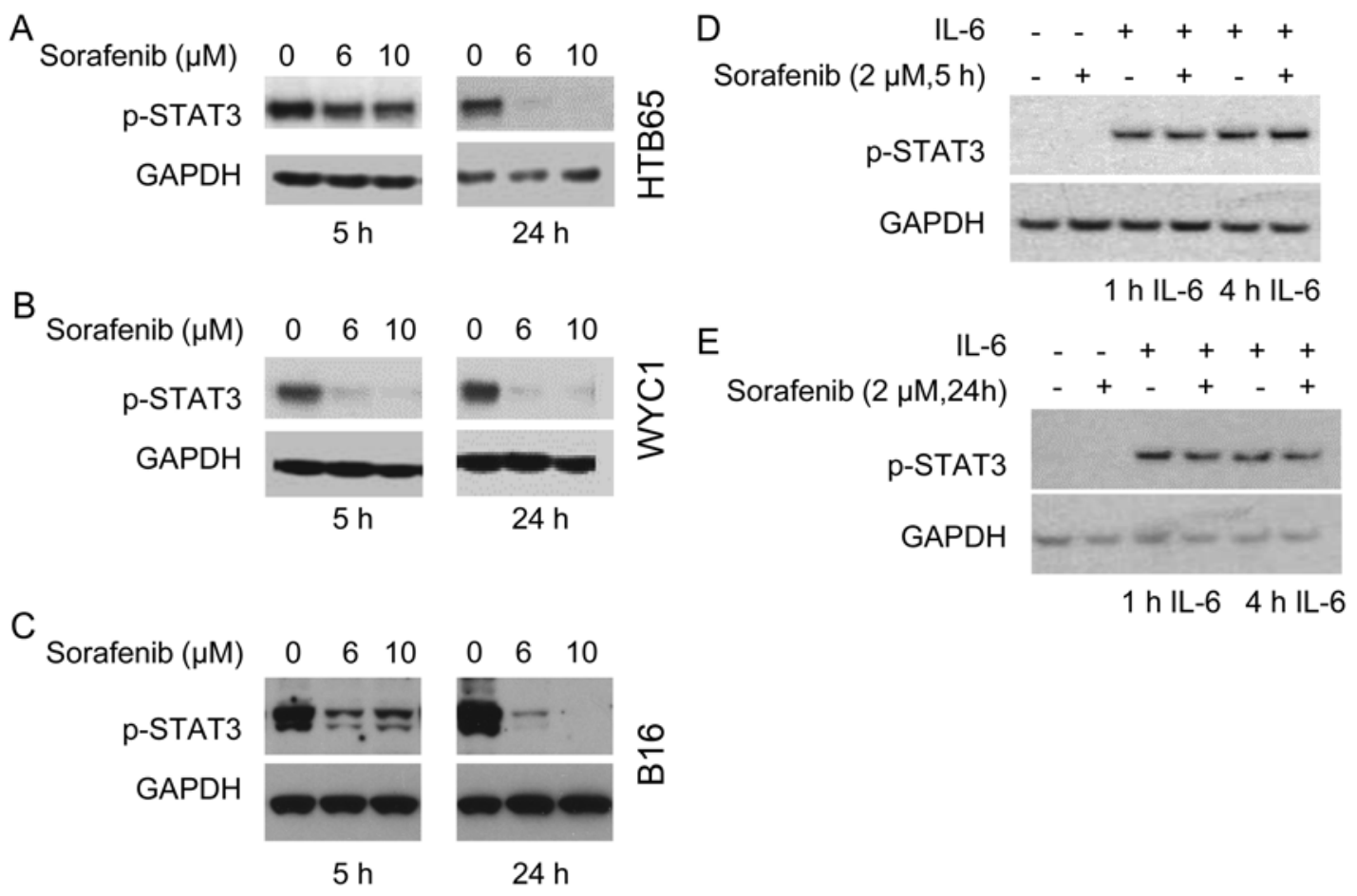

FIG. 2. The phosphorylation status of Stat3 after treatment with sorafenib alone or followed by IL-6 stimulation. Western blot was used to assess the phosphorylation status of Stat3 in HTB65 (A), WYC1 (B), and B16 (C) 5 and 24 hours after treatment with sorafenib. Serum-starved HTB65 cells were exposed to $2 \mu \mathrm{M}$ sorafenib for 5 or 24 hours (D and E) and subsequently stimulated with IL-6 (10 ng/ml) for 1 or 4 hours. GAPDH = glyceraldehyde-3-phosphate-dehydrogenase.

\section{Mcl-1 and Bcl-xl Expression After Sorafenib Treatment}

The antiapoptotic genes $\mathrm{Mcl}-\mathrm{l}$ and $\mathrm{Bcl}-\mathrm{xl}$ are wellknown downstream Stat3 targets. ${ }^{25}$ Western blot to determine expression of these 2 genes was performed in HTB65, WYC1, and B16 cells 5 and 24 hours after sorafenib treatment (Fig. 4). In HTB65 and B16 cells, Mcl-1 was downregulated after 24 hours. In WYC1 cells, downregulation of $\mathrm{Mcl}-\mathrm{I}$ was observed after 5 hours. Contrary to expectations, $B c l-x l$ was not downregulated by sorafenib treatment in either cell line.

\section{Convection-Enhanced Delivery of Sorafenib In Vivo}

Although CED is a very accurate form of microinfusion, a small amount of leakage of the infusate into healthy surrounding brain tissue is unpreventable. Therefore, it was critical to examine normal brain tolerance for directly injected sorafenib for when leakage occurs. Non-tumorbearing C57BL/6 mice that underwent CED of $10 \mu \mathrm{M}$ or $100 \mu \mathrm{M}$ sorafenib into normal brain were observed for up to 1 month for toxicity. The mice tolerated both doses very well, displaying no weight loss, neurological deficits, or behavioral abnormalities (supplementary Table 1). H \& E staining of the brain tissue also revealed no evidence of neurotoxicity (supplementary Fig. 2).

Because CED has not been used in melanomas previously, we then optimized parameters for CED into our intracranial melanoma model, using Evans Blue as a surrogate marker. Distribution of $10 \mu \mathrm{l}$ of Evans Blue into normal brain and melanomas achieved a volume of distri- bution that encompassed the entire tumor (supplementary Fig. 3).

Next, we examined the effect of CED of sorafenib into B16-ffluc+ melanomas implanted intracranially in C57BL/6 mice. Bioluminescent imaging revealed that CED of sorafenib 6 days after implantation decreased the tumor volume compared with CED of the vehicle control (p < 0.05) (Fig. 5A). On Day 3 after treatment, average tumor bioluminescence in animals given sorafenib via
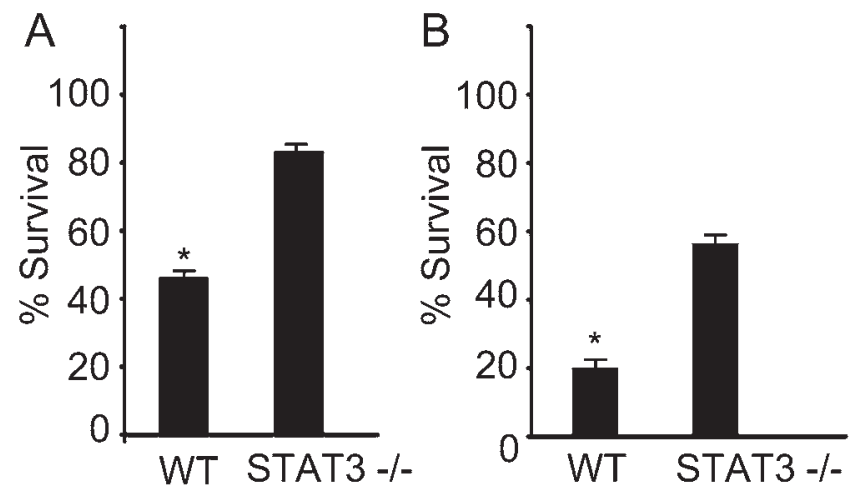

FIG. 3. Sorafenib decreases viability via the Stat3 pathway. Cell viability was assessed in MEF Stat $3+/+$ and MEF Stat $3-/$ - cell lines treated with $6 \mu \mathrm{M}(\mathrm{A})$ or $12 \mu \mathrm{M}(\mathrm{B})$ sorafenib. Cytotoxicity was greater in Stat3 $+/+$ than in Stat $3-/-$ cell lines $(p<0.05)$, suggesting an important role for Stat3 inhibition in sorafenib's tumor-killing ability. WT = wild type $($ Stat $3+/+) ;{ }^{*}=$ significant $(p<0.05)$. 
A

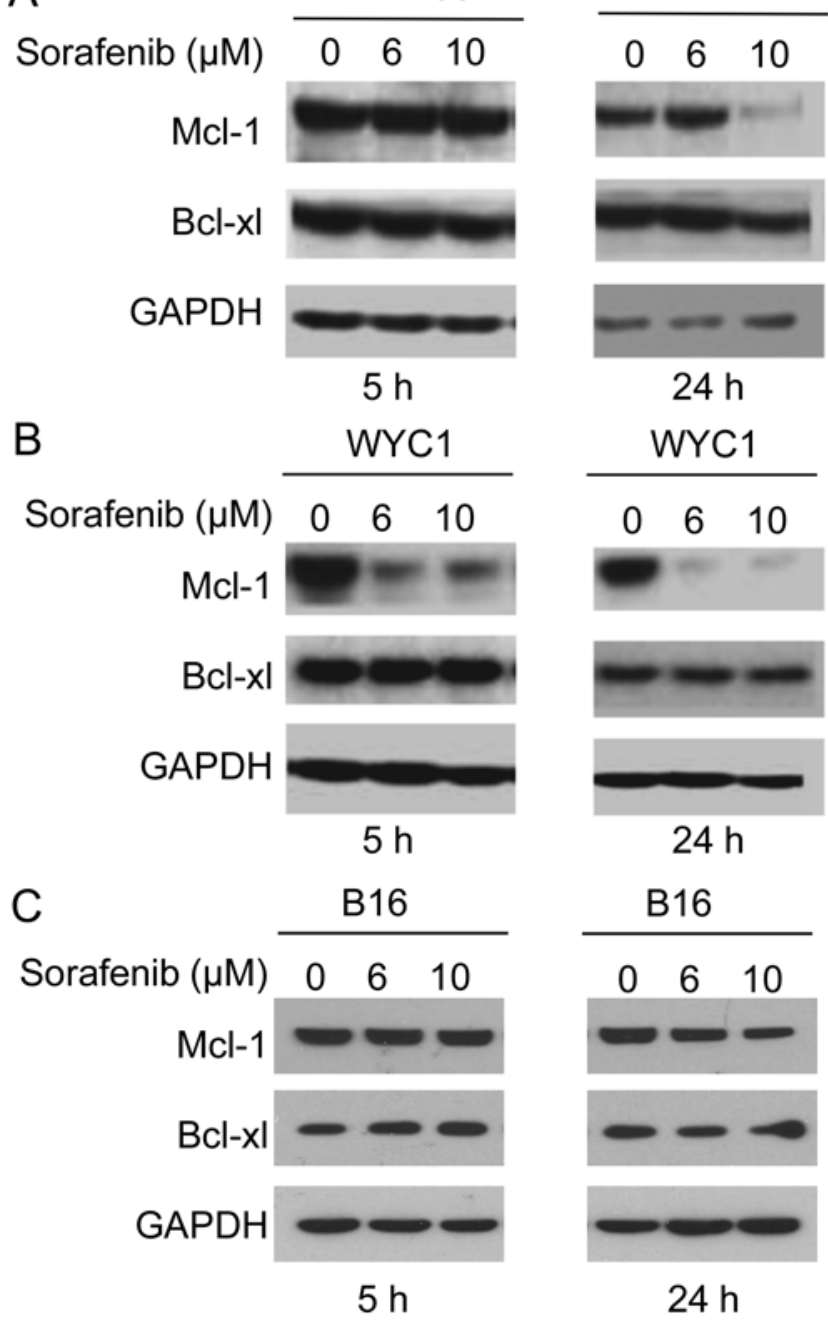

FIG. 4. Downregulation of antiapoptotic Mcl-1 after treatment with sorafenib. $\mathrm{Mcl}-1$ and $\mathrm{BCl}-\mathrm{xl}$ protein expressions were examined in melanoma cell lines 5 and 24 hours after treatment with sorafenib. In HTB65 cells, Mcl-1 was inhibited only at the 24-hour time point, using $10 \mu \mathrm{M}$ sorafenib (A). WYC1 cells showed a more rapid response, in which sorafenib suppressed $\mathrm{Mcl}-1$ within 5 hours at a low dose $(6 \mu \mathrm{M})$ (B). In B16 cells, Mcl-1 was inhibited at the 24-hour time point by $10 \mu \mathrm{M}$ sorafenib (C). Sorafenib had no effect on $\mathrm{Bcl}-\mathrm{xl}$ expression in all 3 cell lines tested.

CED was $(100 \pm 81) \times 10^{4}$ photons/second, whereas average tumor bioluminescence in vehicle controls was $(500$ $\pm 300) \times 10^{4}$ photons/second (Fig. 5C). Correspondingly, the treatment group survived $150 \%$ longer than the control animals ( $<<0.01$, Fig. 5B).

Because a single treatment showed significant antitumor activity, we investigated if sequential treatments in the same animal would further improve results. CED of sorafenib or vehicle control was then performed on Days 4 and 8 after tumor implantation. To highlight the advantages of CED, we added another control group, in which mice were treated with high doses of orally administered sorafenib. Survival of mice given 2 doses of sorafenib via CED was not significantly different than of those given 1 dose (Fig. 6). Survival of mice given orally administered sorafenib was not significantly different than of animals that received CED of the vehicle control.

\section{Discussion}

Treatment of melanoma brain metastasis is hampered by the cancer's intrinsic resistance to therapy and the protection from systemic agents afforded by the blood-brain barrier. The use of CED alleviates these issues. The technique bypasses the blood-brain barrier and simultaneously allows precise delivery of high concentrations of drugs such as sorafenib, which have limited efficacy at lower doses.

Despite its middling potency, sorafenib is an attractive antimelanoma agent because of its molecular targets. These include the Raf kinases, which are highly active in the majority of melanomas. ${ }^{22}$ In our study, we demonstrated that sorafenib also inhibits Stat3, an active pathway in many cancers, but one that has particularly high levels of expression in melanomas. ${ }^{17}$ Perhaps indicative of sorafenib's mechanism of cytotoxicity, Stat 3 activation was inhibited at $6 \mu \mathrm{M}$ (Fig. 2), which is within the range of the IC50s $(4.5-12 \mu \mathrm{M})$ determined in multiple melanoma cell lines (Fig. 1).

Stat3 signaling can increase chemoresistance by preventing apoptosis through positive upregulation of $\mathrm{Mcl}-\mathrm{I}$ and $B c l-x l$ in diverse human tumor cell types. ${ }^{2,8,10,11}$ Previous studies have also indicated that sorafenib can downregulate the expression of Stat $3^{3,35}$ and $\mathrm{Mcl}-1$ in several types of tumor cells. ${ }^{14,26,28,37}$ The effect of sorafenib on these antiapoptotic genes in melanomas has not been previously described. Our results showed that sorafenib treatment reduced Mcl-1, but not Bcl-xl, expression in melanoma cell lines (Fig. 4). This is consistent with previous studies, ${ }^{40}$ which indicate that the relationship between Stat3, Mcl-1, and $B c l-x l$ in melanomas is complex and often contextual. Our data also suggest that inhibition of the Stat3/ Mcl-1 pathway plays a role in sorafenib's cytotoxicity. Further supporting this hypothesis, we found that MEF Stat3 +/+ cells were significantly more sensitive to sorafenib than MEF Stat3 -/- cells (Fig. 3). If Stat3 was not an important target of sorafenib, one would expect that the MEF Stat3 knockouts would have been equally, if not more, susceptible to sorafenib's effects, particularly because Stat3 is a prosurvival factor.

Because sorafenib is able to suppress multiple pathways important to melanoma survival, we hypothesized that there may be synergy with other chemotherapeutic agents. Indeed, sorafenib is synergistic with agents such as vorinostat, sunitinib, and gemcitabine. , $, 27,34$ However, we found no such synergy with either melphalan or temozolomide. Because both of these drugs are alkylating agents, it is possible that the 2 pathways through which these drugs and sorafenib exert cytotoxicity do not cross and could even interfere with one another. For example, some evidence suggests that there may be an antagonistic effect because alkylating agents upregulate $\mathrm{Mcl}-\mathrm{I}$ expression in myeloid leukemia cells. ${ }^{38}$

Because IC50s were in the $4.5-12 \mu \mathrm{M}$ range, this concentration of drugs would be extraordinarily difficult 

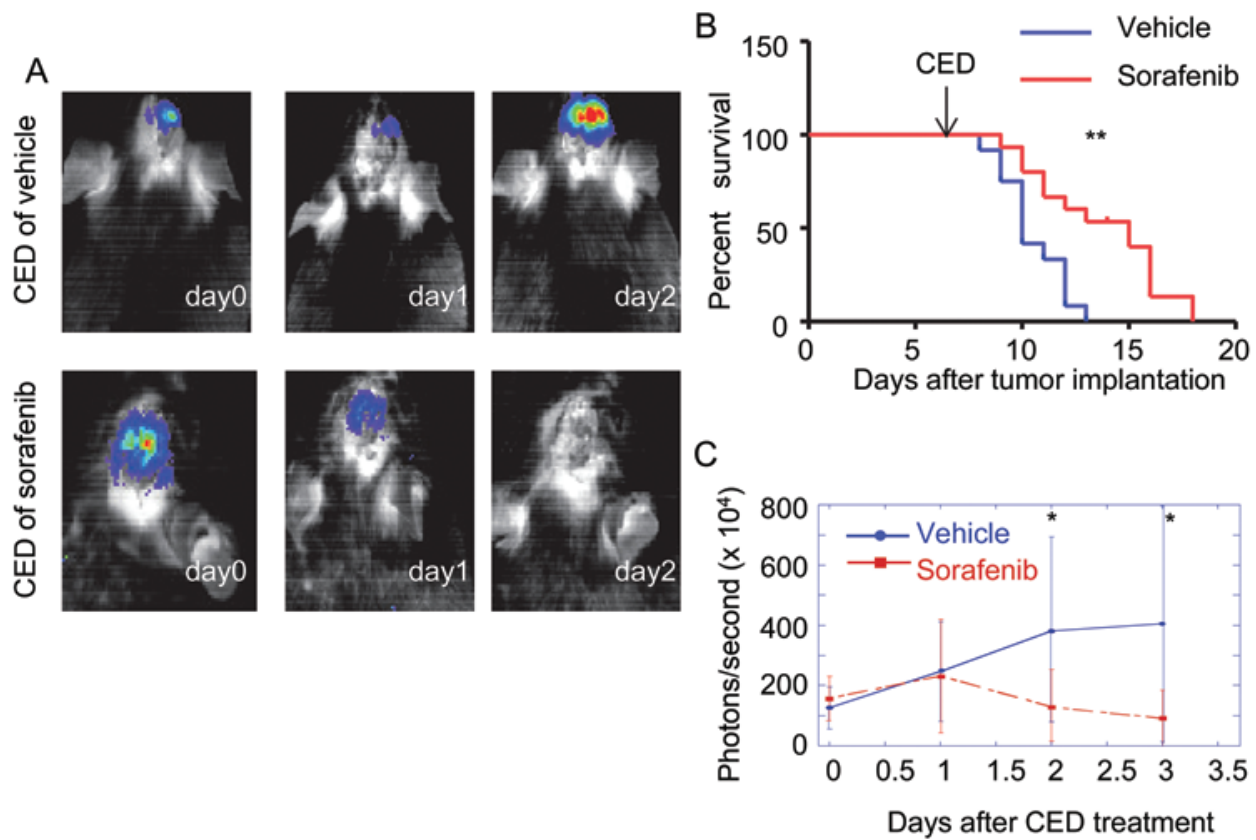

FIG. 5. Regression of established melanoma xenografts after CED of sorafenib. Mice bearing intracranial B16-ffluc tumors were treated by CED of sorafenib or vehicle 6 days after tumor implantation. Bioluminescent imaging of intracranial tumor burden at Days 0,1 , and 2 after treatment show a dramatic effect of CED of sorafenib (A). Kaplan-Meier analysis showed improved survival for the mice treated by CED of sorafenib $\left(15 \pm 2.58\right.$ days) versus vehicle control $(10 \pm 1.51$ days $)\left({ }^{* *} p<0.01 ; n=6-8\right)$ (B). Average bioluminescent signal from tumors in animals treated with sorafenib was significantly decreased compared with animals treated with vehicle $\left({ }^{*} p<0.05 ; n=6-8\right)$ (C).
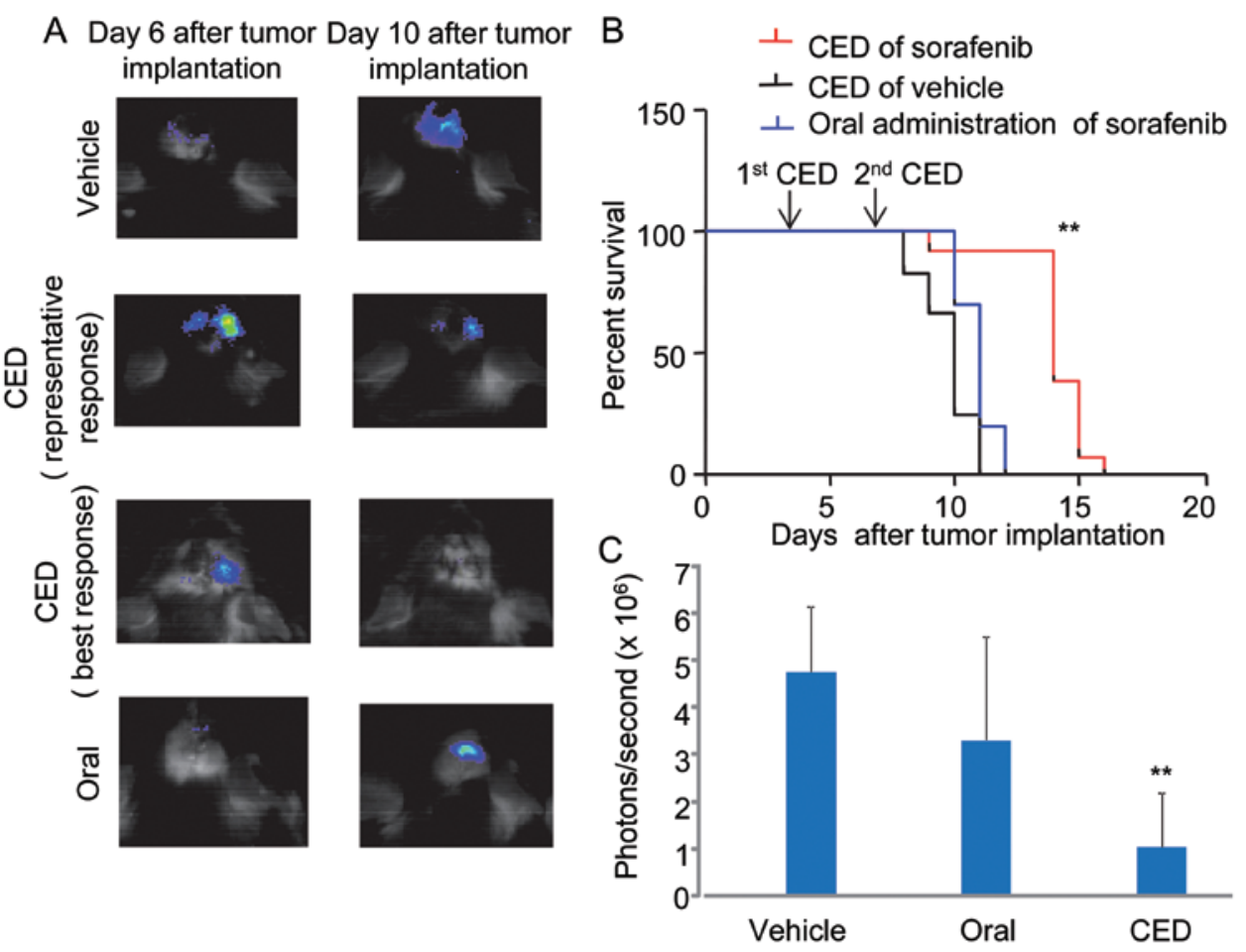

FIG. 6. Effect of serial CED of sorafenib in a murine model of melanoma brain metastasis. CED of sorafenib (100 $\mu \mathrm{M})$ or vehicle control was performed on Days 4 and 8 after B16-ffluc+ implantation. This was compared with a group of animals in which sorafenib was administered orally (50 mg/kg/day) for 7 days. Bioluminescent imaging done on Days 6 and 10 after tumor implantation showed responses to convected and systemically given sorafenib and control (A). Kaplan-Meier analysis showed improved survival for mice that received CED of sorafenib compared with CED of vehicle or oral administration $\left.{ }^{* *} p<0.01 ; n=6-8\right)(B)$. Graph indicating that CED of sorafenib significantly decreased tumor growth $\left({ }^{* *} p<0.01 ; n=6-8\right)(C)$. 
to achieve in any particular tissue, much less the brain. Highlighting this fact is the failure of high doses of orally administered sorafenib to have an effect on tumor size or survival in mice with intracranial melanoma implants (Fig. 6). This contrasts sharply with the animals that were treated by CED of sorafenib. These animals exhibited $150 \%$ greater survival, with corresponding responses seen in noninvasive imaging (Figs. 5 and 6). To improve on this result, we investigated if serial treatments would further prolong survival. Surprisingly, this approach did not cause the desired effect (Fig. 6). We speculated that the cells that were sensitive to sorafenib were already killed by the first treatment, leaving behind cells resistant to the second treatment. Indeed, in clinical practice, rapid tolerance occurs to small-molecule inhibitors used for melanoma therapy.

Overall, our results demonstrate that CED of sorafenib into melanomas within the brain can decrease tumor size and increase survival in a murine model. However, predicting the utility of this technique in the clinical setting is difficult. Convective distribution of a drug in a rodent brain tumor is more readily achievable than in the human counterpart. One of the main underlying causes of the discrepancy is the difference in size between rodent and human brain tumors. Rodent brain tumors are so small that even when convective flow does not achieve ideal drug distribution, diffusion of the drug can still cause a major effect. Human brain tumors are usually up to an order of magnitude larger and thus demand more of the convective, rather than the diffusive, component of CED for drug distribution. Furthermore, the larger target volume introduces more tissue heterogeneity, which adversely affects the predictability of convective distribution. Therefore, effects of CED could be overestimated in a murine model. It is also difficult to estimate side effects of CED of drugs into the brain by using a murine model. Despite these limitations, numerous human studies using CED have confirmed that the fundamental properties of CED established in the murine model remain the same. CED of small-molecule inhibitors appears to be a promising approach warranting further investigation and refinement.

\section{Conclusions}

Our study suggests that sorafenib's inhibition of Stat3 in melanoma cells is one mechanism through which it causes cellular death. Convective delivery of sorafenib is feasible and augments its effectiveness in an intracranial model of melanoma metastasis. In light of the results of recent clinical trials, it would be particularly interesting to investigate CED of more potent antimelanoma smallmolecule inhibitors, such as ipilimumab or vemurafenib.

\section{Acknowledgment}

This project was supported by the National Institutes of Health K12, Clinical Oncology Career Development Grant (CA001727).

\section{References}

1. Aggarwal BB, Kunnumakkara AB, Harikumar KB, Gupta SR, Tharakan ST, Koca C, et al: Signal transducer and activator of transcription-3, inflammation, and cancer: how intimate is the relationship? Ann N Y Acad Sci 1171:59-76, 2009

2. Catlett-Falcone R, Landowski TH, Oshiro MM, Turkson J, Levitzki A, Savino R, et al: Constitutive activation of Stat3 signaling confers resistance to apoptosis in human U266 myeloma cells. Immunity 10:105-115, 1999

3. Chai H, Luo AZ, Weerasinghe P, Brown RE: Sorafenib downregulates ERK/Akt and STAT3 survival pathways and induces apoptosis in a human neuroblastoma cell line. Int $\mathbf{J}$ Clin Exp Pathol 3:408-415, 2010

4. Chen MY, Lonser RR, Morrison PF, Governale LS, Oldfield $\mathrm{EH}$ : Variables affecting convection-enhanced delivery to the striatum: a systematic examination of rate of infusion, cannula size, infusate concentration, and tissue-cannula sealing time. J Neurosurg 90:315-320, 1999

5. Dasmahapatra G, Yerram N, Dai Y, Dent P, Grant S: Synergistic interactions between vorinostat and sorafenib in chronic myelogenous leukemia cells involve Mcl-1 and p21CIP1 down-regulation. Clin Cancer Res 13:4280-4290, 2007

6. Delgado JS, Mustafi R, Yee J, Cerda S, Chumsangsri A, Dougherty U, et al: Sorafenib triggers antiproliferative and pro-apoptotic signals in human esophageal adenocarcinoma cells. Dig Dis Sci 53:3055-3064, 2008

7. Eggermont AM, Robert C: New drugs in melanoma: it's a whole new world. Eur J Cancer 47:2150-2157, 2011

8. Epling-Burnette PK, Liu JH, Catlett-Falcone R, Turkson J, Oshiro M, Kothapalli R, et al: Inhibition of STAT3 signaling leads to apoptosis of leukemic large granular lymphocytes and decreased Mcl-1 expression. J Clin Invest 107:351-362, 2001

9. Fu T, He Q, Sharma P: The ICOS/ICOSL pathway is required for optimal antitumor responses mediated by anti-CTLA-4 therapy. Cancer Res 71:5445-5454, 2011

10. Grandis JR, Drenning SD, Zeng Q, Watkins SC, Melhem MF, Endo S, et al: Constitutive activation of Stat3 signaling abrogates apoptosis in squamous cell carcinogenesis in vivo. Proc Natl Acad Sci U S A 97:4227-4232, 2000

11. Gritsko T, Williams A, Turkson J, Kaneko S, Bowman T, Huang M, et al: Persistent activation of stat3 signaling induces survivin gene expression and confers resistance to apoptosis in human breast cancer cells. Clin Cancer Res 12:11-19, 2006

12. Hata Y, Sandler A, Loehrer PJ, Sledge GW Jr, Weber G: Synergism of taxol and gallium nitrate in human breast carcinoma cells: schedule dependency. Oncol Res 6:19-24, 1994

13. Jing N, Tweardy DJ: Targeting Stat3 in cancer therapy. Anticancer Drugs 16:601-607, 2005

14. Katz SI, Zhou L, Chao G, Smith CD, Ferrara T, Wang W, et al: Sorafenib inhibits ERK1/2 and MCL-1(L) phosphorylation levels resulting in caspase-independent cell death in malignant pleural mesothelioma. Cancer Biol Ther 8:2406-2416, 2009

15. Laske DW, Morrison PF, Lieberman DM, Corthesy ME, Reynolds JC, Stewart-Henney PA, et al: Chronic interstitial infusion of protein to primate brain: determination of drug distribution and clearance with single-photon emission computerized tomography imaging. J Neurosurg 87:586-594, 1997

16. Lieberman DM, Laske DW, Morrison PF, Bankiewicz KS, Oldfield EH: Convection-enhanced distribution of large molecules in gray matter during interstitial drug infusion. J Neurosurg 82:1021-1029, 1995

17. Messina JL, Yu H, Riker AI, Munster PN, Jove RL, Daud AI: Activated stat-3 in melanoma. Cancer Contr 15:196-201, 2008

18. Morrison PF, Chen MY, Chadwick RS, Lonser RR, Oldfield $\mathrm{EH}$ : Focal delivery during direct infusion to brain: role of flow rate, catheter diameter, and tissue mechanics. Am J Physiol 277:R1218-R1229, 1999 
19. Morrison PF, Laske DW, Bobo H, Oldfield EH, Dedrick RL: High-flow microinfusion: tissue penetration and pharmacodynamics. Am J Physiol 266:R292-R305, 1994

20. Natarajan N, Telang S, Miller D, Chesney J: Novel immunotherapeutic agents and small molecule antagonists of signalling kinases for the treatment of metastatic melanoma. Drugs 71:1233-1250, 2011

21. Niu G, Bowman T, Huang M, Shivers S, Reintgen D, Daud A, et al: Roles of activated Src and Stat3 signaling in melanoma tumor cell growth. Oncogene 21:7001-7010, 2002

22. Omholt K, Grafström E, Kanter-Lewensohn L, Hansson J, Ragnarsson-Olding BK: KIT pathway alterations in mucosal melanomas of the vulva and other sites. Clin Cancer Res 17:3933-3942, 2011

23. Ott PA, Hamilton A, Min C, Safarzadeh-Amiri S, Goldberg L, Yoon J, et al: A phase II trial of sorafenib in metastatic melanoma with tissue correlates. PLoS One 5:e15588, 2010

24. Panka DJ, Wang W, Atkins MB, Mier JW: The Raf inhibitor BAY 43-9006 (sorafenib) induces caspase-independent apoptosis in melanoma cells. Cancer Res 66:1611-1619, 2006

25. Quintanilla-Martinez L, Kremer M, Specht K, Calzada-Wack J, Nathrath M, Schaich R, et al: Analysis of signal transducer and activator of transcription 3 (Stat 3) pathway in multiple myeloma: Stat 3 activation and cyclin D1 dysregulation are mutually exclusive events. Am J Pathol 162:1449-1461, 2003

26. Rahmani M, Davis EM, Bauer C, Dent P, Grant S: Apoptosis induced by the kinase inhibitor BAY 43-9006 in human leukemia cells involves down-regulation of Mcl-1 through inhibition of translation. J Biol Chem 280:35217-35227, 2005

27. Ricciardi S, Mey V, Nannizzi S, Pasqualetti G, Crea F, Del Tacca M, et al: Synergistic cytotoxicity and molecular interaction on drug targets of sorafenib and gemcitabine in human pancreas cancer cells. Chemotherapy 56:303-312, 2010

28. Rosato RR, Almenara JA, Coe S, Grant S: The multikinase inhibitor sorafenib potentiates TRAIL lethality in human leukemia cells in association with Mcl-1 and cFLIPL downregulation. Cancer Res 67:9490-9500, 2007

29. Sloan AE, Nock CJ, Einstein DB: Diagnosis and treatment of melanoma brain metastasis: a literature review. Cancer Contr 16:248-255, 2009

30. Smalley KS, Xiao M, Villanueva J, Nguyen TK, Flaherty KT, Letrero R, et al: CRAF inhibition induces apoptosis in melanoma cells with non-V600E BRAF mutations. Oncogene 28:85-94, 2009

31. Tai WT, Cheng AL, Shiau CW, Huang HP, Huang JW, Chen PJ, et al: Signal transducer and activator of transcription 3 is a major kinase-independent target of sorafenib in hepatocellular carcinoma. J Hepatol 55:1041-1048, 2011

32. Turley RS, Raymond AK, Tyler DS: Regional treatment strategies for in-transit melanoma metastasis. Surg Oncol Clin N Am 20:79-103, 2011

33. Valles F, Fiandaca MS, Eberling JL, Starr PA, Larson PS, Christine CW, et al: Qualitative imaging of adeno-associated virus serotype 2-human aromatic L-amino acid decarboxylase gene therapy in a phase I study for the treatment of Parkinson disease. Neurosurgery 67:1377-1385, 2010

34. Vogl UM, Berger W, Micksche M, Pirker C, Lamm W, Pichelmeyer O, et al: Synergistic effect of sorafenib and suni- tinib with enzastaurin, a selective protein kinase $\mathrm{C}$ inhibitor in renal cell carcinoma cell lines. Cancer Lett 277:218-226, 2009

35. Yang F, Van Meter TE, Buettner R, Hedvat M, Liang W, Kowolik CM, et al: Sorafenib inhibits signal transducer and activator of transcription 3 signaling associated with growth arrest and apoptosis of medulloblastomas. Mol Cancer Ther 7:3519-3526, 2008

36. Yang L, Ma X, Xiao L, Tang M, Weng X, Sun L, et al: Uniquely modified RNA oligonucleotides targeting STAT3 suppress melanoma growth both in vitro and in vivo. Cancer Biol Ther 8:2065-2072, 2009

37. Yu C, Bruzek LM, Meng XW, Gores GJ, Carter CA, Kaufmann SH, et al: The role of Mcl-1 downregulation in the proapoptotic activity of the multikinase inhibitor BAY 439006. Oncogene 24:6861-6869, 2005

38. Zhan Q, Bieszczad CK, Bae I, Fornace AJ Jr, Craig RW: Induction of BCL2 family member MCL1 as an early response to DNA damage. Oncogene 14:1031-1039, 1997

39. Zhang L, Lau YK, Xia W, Hortobagyi GN, Hung MC: Tyrosine kinase inhibitor emodin suppresses growth of HER-2/ neu-overexpressing breast cancer cells in athymic mice and sensitizes these cells to the inhibitory effect of paclitaxel. Clin Cancer Res 5:343-353, 1999

40. Zhuang L, Lee CS, Scolyer RA, McCarthy SW, Zhang XD, Thompson JF, et al: Mcl-1, Bcl-XL and Stat 3 expression are associated with progression of melanoma whereas Bcl-2, AP-2 and MITF levels decrease during progression of melanoma. Mod Pathol 20:416-426, 2007

\section{Disclosure}

The authors report no conflict of interest concerning the materials or methods used in this study or the findings specified in this paper.

\section{Author Contributions}

Conception and design: Chen, Zou, Yang, Jove, Li. Acquisition of data: Zou, Yin, Hsu, Li, Brandon. Analysis and interpretation of data: Chen, Zou, Li. Drafting the article: Chen, Zou, Li. Critically revising the article: Chen, Jandial, Li. Reviewed submitted version of manuscript: Chen, Lin. Approved the final version of the manuscript on behalf of all authors: Chen. Statistical analysis: Chen, Zou. Administrative/technical/material support: Chen. Study supervision: Chen, Li.

\section{Supplemental Information \\ Online-Only Content}

Supplemental material is available with the online version of the article.

Supplementary Table 1 and Supplementary Figures 1-3. http://thejns.org/doi/suppl/10.3171/2015.3.JNS132040.

\section{Correspondence}

Mike Chen, Division of Neurosurgery, City of Hope National Medical Center, MOB 2001J, 1500 East Duarte Rd., Duarte, CA 91010.email: mchen@coh.org. 